\title{
Elliptical torii in a constant magnetic field
}

\author{
M. Encinosa* and M. Jack \\ Florida A\&M University \\ Department of Physics \\ Tallahassee FL 32307
}

\begin{abstract}
The Schrodinger equation for an electron on the surface of an elliptical torus in the presence of a constant azimuthally symmetric magnetic field is developed. The single particle spectrum and eigenfunctions as a function of magnetic flux through the torus are determined and it is shown that inclusion of the geometric potential is necessary to recover the limiting cases of vertical strip and flat ring structures.
\end{abstract}

PACS numbers: 03.65Ge,03.65-w 68.65.-k

Keywords: elliptical, torus, magnetic field

*Electronic address: encinosa@cennas.nhmfl.gov 


\section{INTRODUCTION}

Toroidal nanostructures present intriguing possibilites for use as nano-device elements

1, 2, 3, 4]. In addition to Aharanov-Bohm and persistent azimuthal current effects known to exist in quantum rings [5, 6, ㄱ, 8, ㅁ, 10, 11], toroidal structures allow for motions around the minor radius of the torus subject to boundary conditions distinct from those for flat rings (Figs. 1-3).

For hollow torii, electrons are thought to be localized near the surface of the object. The restriction to motion near a surface has interesting manifestations. Recent work [12, 13] on a torus of major radius $R$ and circular cross section of radius $a$, has shown that a surface dependent geometric potential $V_{C}$ [14, 15, 16, 17, 18, 19] is important even for electrons that can wander substantial distances away from the surface and should be employed as an effective potential when considering two dimensional problems on curved surfaces. Curvature effects are more pronounced for elliptical torii $\left(E T^{2}\right)$ which can behave in different ways than a torus with circular cross section. A recent paper by Gravesen, Willatzen and Lew Yan Voon in this journal [20] dealt with particles constrained to motion on surfaces of revolution, including elliptical torii. There the influence of toroidal eccentricity and $V_{C}$ were shown to strongly affect system eigenvalues and eigenfunctions. This work is concerned with the extension of 20] to include a constant magnetic field along the z-axis, and the role $V_{C}$ plays in recovering the ribbon (taken to be an infinitely thin vertical strip of radius $R$ and height $2 b$ ) and flat ring (an annular region of inner radius $R-a$ and outer radius $R+a$ ) limits.

This paper is organized as follows: section 2 presents the geometry leading to, and the formalism by which, the Hamiltonian on $E T^{2}$ is derived inclusive of a magnetic field $\mathbf{B}=B_{0} \mathbf{e}_{z}$. The methodology presented in section 2 differs from that given in [20] because here the magnetic field is to be incorporated via the minimal prescription so it proves advantageous to first derive the gradient operator rather than directly employ

$$
\nabla^{2}=g^{-\frac{1}{2}} \frac{\partial}{\partial q^{i}}\left[g^{\frac{1}{2}} g^{i j} \frac{\partial}{\partial q^{j}}\right]
$$

In section 3 numerical results are given as curves of single particle ground state energies $\varepsilon_{0}$ as functions of magnetic flux $\gamma$ for elliptical torii of several eccentricities. Section 4 is reserved for conclusions and suggestions for future work. 


\section{DEVELOPMENT OF THE HAMILTONIAN ON $E T^{2}$}

As noted above, including a vector potential $\mathbf{A}$ in the Hamiltonian makes it advantageous to derive the gradient operator and proceed to include $\mathbf{A}$ by the minimal prescription.

Let $\left(\mathbf{e}_{\rho}, \mathbf{e}_{\phi}, \mathbf{e}_{z}\right)$ be cylindrical coordinate system unit vectors. Parameterize points near an elliptical toroidal surface of major radius $R$ and minor radii $a$ and $b$ by

$$
\mathbf{x}(\theta, \phi, q)=(R+a \cos \theta) \mathbf{e}_{\rho}+b \sin \theta \mathbf{e}_{z}+q \mathbf{e}_{n}
$$

with $\mathbf{e}_{n}$ the unit normal to $E T^{2}$ to be defined momentarily and $q$ the coordinate measuring the distance from the surface. In what follows the major radius $R$ will be set to $R=500 \AA$, which is in accordance with fabricated structures [4, 21, 22]. From Eq. (2)

$$
d \mathbf{x}=P d \theta \mathbf{e}_{1}+W d \phi \mathbf{e}_{\phi}+d q \mathbf{e}_{n}+q d \mathbf{e}_{n}
$$

with

$$
\begin{gathered}
P=\left(a^{2} \sin ^{2} \theta+b^{2} \cos ^{2} \theta\right)^{1 / 2} \\
W=1+\alpha \cos \theta \\
\mathbf{e}_{1}=\frac{1}{P}\left(-a \sin \theta \mathbf{e}_{\rho}+b \cos \theta \mathbf{e}_{z}\right)
\end{gathered}
$$

and

$$
\mathbf{e}_{n}=\frac{1}{P}\left(a \sin \theta \mathbf{e}_{z}+b \cos \theta \mathbf{e}_{\rho}\right)
$$

The unit vectors $\left(\mathbf{e}_{1}, \mathbf{e}_{n}\right)$ are tangent to $E T^{2}$ in the direction of increasing $\theta$ and $\phi$ respectively.

From

$$
d \mathbf{e}_{n}=\frac{a b}{P^{2}} \mathbf{e}_{1} d \theta+\frac{b}{P} \cos \theta \mathbf{e}_{\phi} d \phi,
$$

the vector differential line element can be found from Eqs. (8) and (3), leading to a gradient operator

$$
\nabla=\frac{1}{P\left(1+k_{\theta} q\right)} \mathbf{e}_{1} \frac{\partial}{\partial \theta}+\frac{1}{W\left(1+k_{\phi} q\right)} \mathbf{e}_{\phi} \frac{\partial}{\partial \phi}+\mathbf{e}_{q} \frac{\partial}{\partial q}
$$

with $k_{\theta}, k_{\phi}$ the principle curvatures given by

$$
k_{\theta}=\frac{a b}{P^{3}}, \quad k_{\phi}=\frac{b \cos \theta}{W P} .
$$


The vector potential (working in the Coulomb $\nabla \cdot \mathbf{A}=0$ gauge) appropriate to $B_{0} \mathbf{e}_{z}$ is

$$
\mathbf{A}=\frac{B_{0}}{2}\left(W+\frac{q b \cos \theta}{P}\right) \mathbf{e}_{\phi}
$$

The Schrodinger equation $(\hbar=m=1)$

$$
\frac{1}{2}\left(\frac{1}{i} \nabla+q \boldsymbol{A}\right)^{2} \Psi=E \Psi
$$

which results from Eqs. (9), (11) and (12) can be reduced to a concise and dimensionless form by first

a. noting

$$
\begin{gathered}
\frac{\partial \mathbf{e}_{1}}{\partial \theta}=-\frac{a b}{P^{2}} \mathbf{e}_{n} \\
\frac{\partial \mathbf{e}_{n}}{\partial \theta}=+\frac{a b}{P^{2}} \mathbf{e}_{1} \\
\frac{\partial \mathbf{e}_{1}}{\partial \phi}=-\frac{a \sin \theta}{P} \mathbf{e}_{\phi} \\
\frac{\partial \mathbf{e}_{n}}{\partial \phi}=+\frac{b \cos \theta}{P} \mathbf{e}_{\phi},
\end{gathered}
$$

b. setting $\alpha=a / R, \beta=b / R, \varepsilon=2 E a^{2}$,

c. letting $D(\theta)=P(\theta) / R, p(\theta)=P(\theta) / a$ and $F(\theta)=1+\alpha \cos \theta$,

d. defining $\gamma=.263 B_{0}$, with $B_{0}$ in Teslas, which is the conversion factor for an $R=500 \AA$ torus,

e. performing the well-known procedure for obtaining $V_{C}$ (which will appear below as the scaled dimensionless function $U_{C}$ ) for which the reader is directed to the relevant references [14, 15, 16, 17, 18, 19, 23, 24, 25, 26, 27, 28, 29, 30],

f. noting that the azimuthal symmetry of the problem allows for the eigenfunction on $E T^{2}$ to be taken as $\Psi(\theta, \phi)=\psi(\theta) \exp [i \nu \phi]$.

Applying the conventions and procedures listed above results in a pair of equations for the surface and normal variables in the $q \rightarrow 0$ limit

$$
\frac{\partial^{2} \psi}{\partial \theta^{2}}-\left(\frac{\alpha \sin \theta}{F(\theta)}+\frac{\alpha^{2}-\beta^{2}}{D^{2}(\theta)}\right) \frac{\partial \psi}{\partial \theta}-\left[\frac{D^{2}(\theta) \nu^{2}}{F^{2}(\theta)}+U_{C}(\theta)+\frac{\gamma^{2} F^{2}(\theta) \alpha^{2} p^{2}(\theta)}{4}+\gamma \nu \alpha^{2} p(\theta)-\varepsilon\right] \psi=0
$$




$$
-\frac{1}{2} \frac{\partial^{2} \chi_{n}}{\partial q^{2}}+V_{n}(q) \chi_{n}=E_{n} \chi_{n} .
$$

The scaled dimensionless curvature potential $U_{C}$ appearing in Eq. (17) is

$$
U_{C}(\theta)=-\frac{1}{4}\left(\frac{\alpha^{2} \beta^{2}}{D^{4}(\theta)}+\frac{\beta^{2} \cos ^{2} \theta}{F^{2}(\theta)}\right) .
$$

The normal confining potential $V_{n}(q)$ can be chosen to take any convenient form, and it is apparent in the $q \rightarrow 0$ limit that the surface Schrodinger equation is independent of the choice. The independence of surface observables on $V_{n}(q)$ has also been shown to be a good approximation when the particle is allowed to move in a finite thin layer by a basis set calculation in the full three dimensional space [13]. The results that follow will not include $E_{n}$; it was also shown in [13] there is negligible state mixing in the $q$ degree of freedom so that the surface spectrum is essentially independent of $E_{n}$.

\section{NUMERICAL RESULTS}

The Schrodinger equation given by Eq. (17) is invariant under $\theta \rightarrow-\theta$ making it possible to separate its eigenfunctions into even and odd $\theta$-parity states by proper choice of

initial conditions at $\theta=0[31]$. Numerically it proves convenient to generate two linearly independent solutions of Eq. (17) with initial conditions

$$
\begin{aligned}
& \psi(0)=1, \psi^{\prime}(0)=0 \rightarrow \psi_{A} \\
& \psi(0)=0, \psi^{\prime}(0)=1 \rightarrow \psi_{B}
\end{aligned}
$$

and insisting upon

$$
\begin{aligned}
& A \psi_{A}(0)+B \psi_{B}(0)=A \psi_{A}(2 \pi)+B \psi_{B}(2 \pi) \\
& A \psi_{A}^{\prime}(0)+B \psi_{B}^{\prime}(0)=A \psi_{A}^{\prime}(2 \pi)+B \psi_{B}^{\prime}(2 \pi) .
\end{aligned}
$$

Eqs. (22) and (23) can be rearranged into a homogeneous linear system for the coefficients $A, B$ and the entire spectrum quickly determined by a simple loop over $\varepsilon$ appearing in Eq. (17). 
Single particle ground state energy $\varepsilon_{0}$ plots as a function of $\gamma$ are given for three toroidal eccentricities in Figs. (4-6). The most interesting feature emerging from inspection of Figs. (4) and (5) is the necessity of including $U_{C}$ to approach the ring and ribbon limit. However, as shown in Figs. (7) and (8), the ring and ribbon limits do differ in detail when compared to the curves for the elliptical torii; because persistent currents are sensitive to the shape of the free energy as a function of flux, the differences in detail may prove important upon a more extensive calculation of spectra. Comparison of the curves shown in Fig. (6) with the values of the limiting cases appearing in the previous two figures indicate clearly that a torus with circular cross-section cannot realistically be approximated with a ring or ribbon, and that the $U_{C}$ alters the structure of $\varepsilon_{0}(\gamma)$ substantially.

\section{CONCLUSIONS}

In this work single-particle ground state energies $\varepsilon_{0}$ as a function of magnetic flux $\gamma$ were calculated for three elliptical torii of very different character. The curves indicate that any attempt to model toroidal structures by approximating them as two-dimensional ribbons or rings must include the geometric potential in order to recover the respective two dimensional limits. This result is surprising in that $V_{C}$ is large only near the regions of substantial curvature and is negligible over most of the structure. Nevertheless, it has considerable effect on $E T^{2}$ eigenvalues, and its omission leads to disagreement with the flat limits. The inclusion of $V_{C}$, however, does not simply trivially reproduce the flat limits for the toroidal eccentricities investigated here; the $\varepsilon_{0}(\gamma)$ curves show differing peak heights and locations than those of the limiting cases.

An interesting natural extension of this work is the addition of an off-axis component of the applied magnetic field. Those cases are perhaps best suited to a basis set method as that employed in [32] subject to modification of the integration measure arising from toroidal eccentricity. The interplay of the magnetic field with regions of substantial curvature may yield interesting mixing of azimuthal modes depending on the magnetic field orientation. 


\section{ACKNOWLEDGEMENTS}

M.E. would like to thank B. Etemadi for useful discussions.

[1] H.R.Shea, R. Martel, and P. Avouris, Phys. Rev. Lett. 84, 4441 (2000).

[2] K. Sasaki, Y. Kawazoe, and R. Saito, Phys. Rev. A 321, 369 (2004).

[3] M. Sano, A. Kamino, J. Okamura, and S. Shinkai, Science 293, 1299 (2003).

[4] H. Zhang, S. W. Chung, and C. A. Mirkin, Nano. Lett. 3, 43 (2003).

[5] I. Filikhin, E. Deyneka, and B. Vlahovic, Modelling Simul. Mater. Sci. Eng. 12, 1121 (2004).

[6] S. Gylfadottir, M. Nita, V. Gudmundsson, and A. Manolescu, Phys. E 27, 209 (2005).

[7] S. Viefers, P. Koskinen, P. S. Deo, and M. Manninen, cond-mat /0310064.

[8] S. Latil, S. Roche, and A. Rubio, Phys. Rev. B 67, 165420 (2003).

[9] K. Sasaki and Y. Kawazoe, Prog. Theo. Phys. 112, 369 (2004).

[10] D. Gridin and A. Adamou, Phys. Rev. B, volume = 69, pages = "155317" (2004).

[11] Y. V. Pershin and C. Piermarocchi, cond-mat/0506187.

[12] M. Encinosa and L.Mott, Phys. Rev. A 68, 014102 (2003).

[13] M. Encinosa, L.Mott, and B. Etemadi, Phys. Scr. 72, 13 (2005).

[14] H. Jensen and H. Koppe, Ann. of Phys. 63, 586 (1971).

[15] R. C. T. da Costa, Phys. Rev. A 23, 1982 (1981).

[16] R. C. T. da Costa, Phys. Rev. A 25, 2893 (1982).

[17] P. Exner and P. Seba, J. Math. Phys. 30, 2574 (1989).

[18] S. Matusani, J. Phys. Soc. Jap. 61, 55 (1991).

[19] M. Burgess and B. Jensen, Phys. Rev. A 48, 1861 (1993).

[20] J. Gravesen, M. Willatzen, and L. L. Y. Voon, Phys. Scr. 72, 105 (2005).

[21] A. Lorke, R. J. Luyken, A. O. Govorov, and J. P. Kotthaus, Phys. Rev. Lett 84, 2223 (2000).

[22] J. M. Garca, G. Medeiros-Ribeiro, K. Schmidt, T. Ngo, J. L. Feng, A. Lorke, J. Kotthaus, and P. M. Petroff, App. Phys. Lett. 71, 2014 (1997).

[23] P. Duclos and P. Exner, Rev. Math. Phys. 7, 73 (1995).

[24] J. Londergan, J. Carini, and D. Murdock, Binding and scattering in two dimensional systems; applications to quantum wires, waveguides, and photonic crystals (Springer-Verlag, Berlin, 
1999).

[25] J. Goldstone and R. L. Jaffe, Phys. Rev. B 45, 14100 (1991).

[26] P. Ouyang, V. Mohta, and R. L. Jaffe, Ann. of Phys. 275, 297 (1998).

[27] I. Popov, Phys. Lett. A 269, 148 (2000).

[28] S. Midgley and J. Wang, Aus. J. Phys. 53, 77 (2000).

[29] I. J. Clark and A. J. Bracken, J. Phys. A 29, 4527 (1996).

[30] P. C. Schuster and R. L. Jaffe, Ann. Phys. 307, 132 (2003).

[31] M. Encinosa and B. Etemadi, Found. Phys. Lett. 16, 403 (2003).

[32] M. Encinosa, Phys. E 28, 209 (2005). 


\section{Figure Captions}

Figs. 1-3: Bohmian trajectories corresponding to a two eigenfunctions superposition on an $R=500 \AA$ A,$a=250 \AA$ torus with a circular cross section as a function of magnetic field values (top to bottom) $B_{0}=0,2,4$ Tesla.

Fig. 4: $\varepsilon_{0}$ for an $\alpha=.5, \beta=.1$ elliptical torus plotted as a function of magnetic flux $\gamma=.263 B_{0}$. Diamonds correspond to the $U_{C}=0$ case, stars to $U_{C} \neq 0$, and squares to a two dimensional annular region with inner radius $1-\alpha$ and outer radius $1+\alpha$.

Fig. 5: $\varepsilon_{0}$ for an $\alpha=.1, \beta=.5$ elliptical torus plotted as a function of magnetic flux $\gamma=.263 B_{0}$. Diamonds correspond to the $U_{C}=0$ case, stars to $U_{C} \neq 0$, and squares to a two dimensional vertical strip/ribbon with unit radius and height $2 \beta$.

Fig. 6: $\varepsilon_{0}$ for an $\alpha=.5$ circular torus plotted as a function of magnetic flux $\gamma=.263 B_{0}$. Diamonds correspond to the $U_{C}=0$ case, stars to $U_{C} \neq 0$.

Fig. 7: Detailed plot of $\varepsilon_{0}$ for an $\alpha=.5, \beta=.1$ elliptical torus plotted as a function of magnetic flux $\gamma=.263 B_{0}$. Stars correspond to the limit of the two-dimensional annular region with inner and outer radii $1 \mp \alpha$ and diamonds to the elliptical torus with $U_{C} \neq 0$.

Fig. 8: Detailed plot of $\varepsilon_{0}$ for an $\alpha=.1, \beta=.5$ elliptical torus plotted as a function of magnetic flux $\gamma=.263 B_{0}$. Stars correspond to the limit of the two-dimensional vertical strip/ribbon of unit radius and height $2 \beta$, and diamonds to the elliptical torus with $U_{C} \neq 0$. 

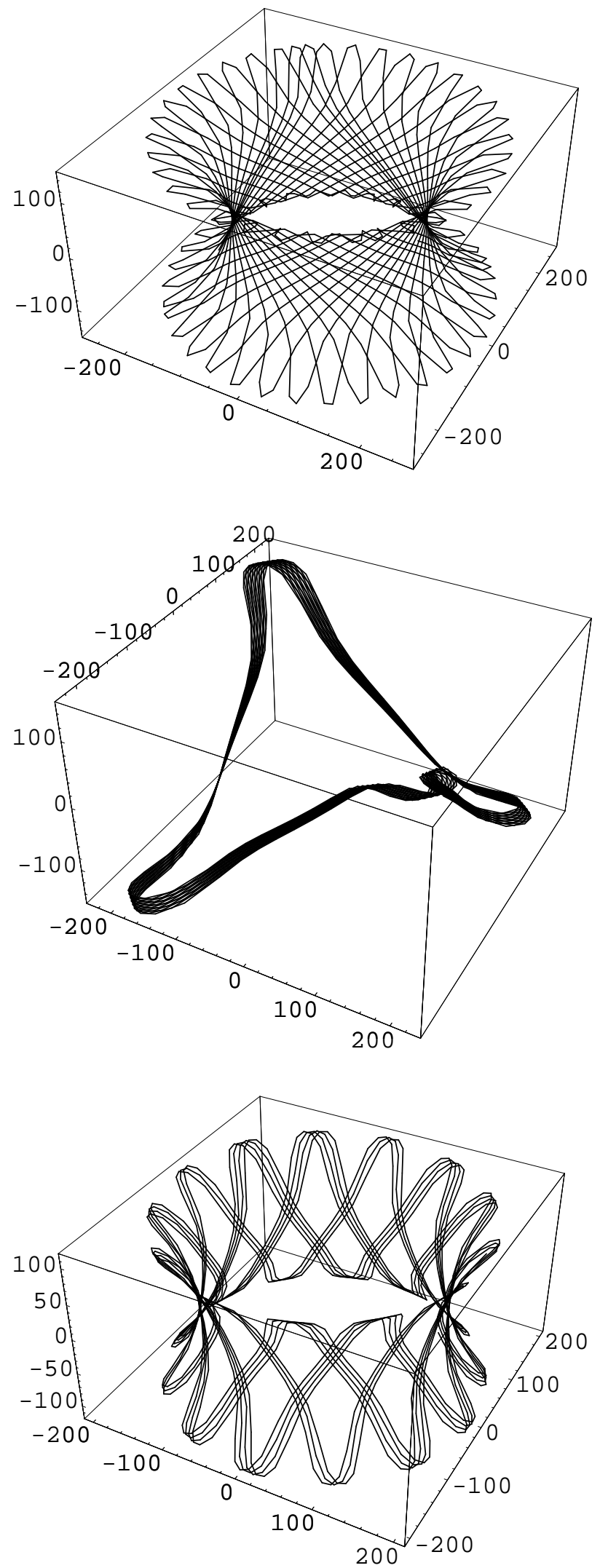

Figs. 1-3 


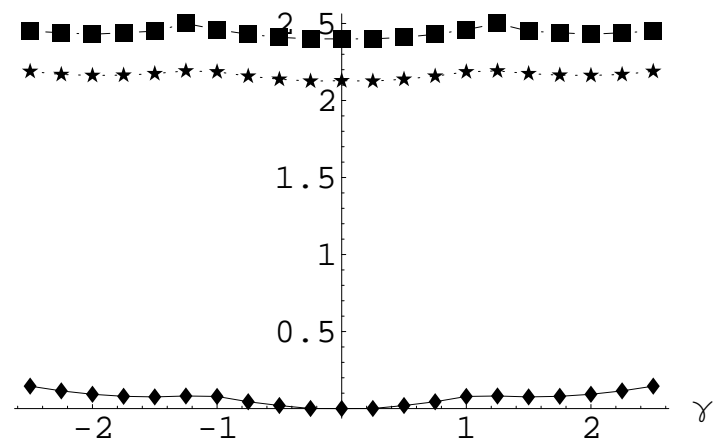

Fig. 4

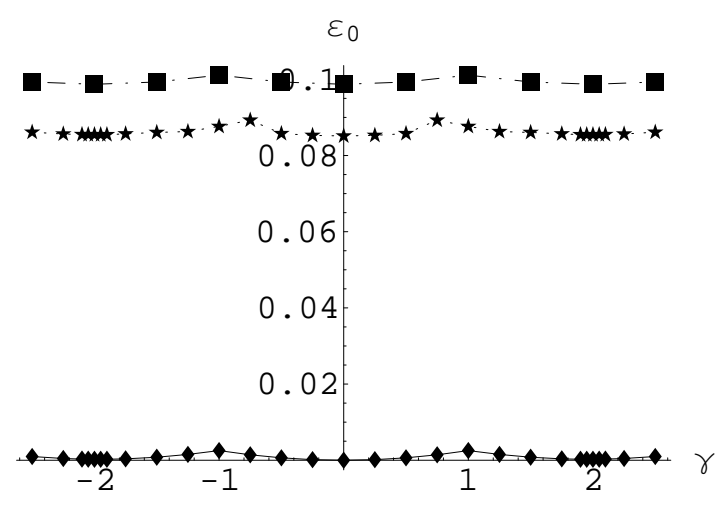

Fig. 5

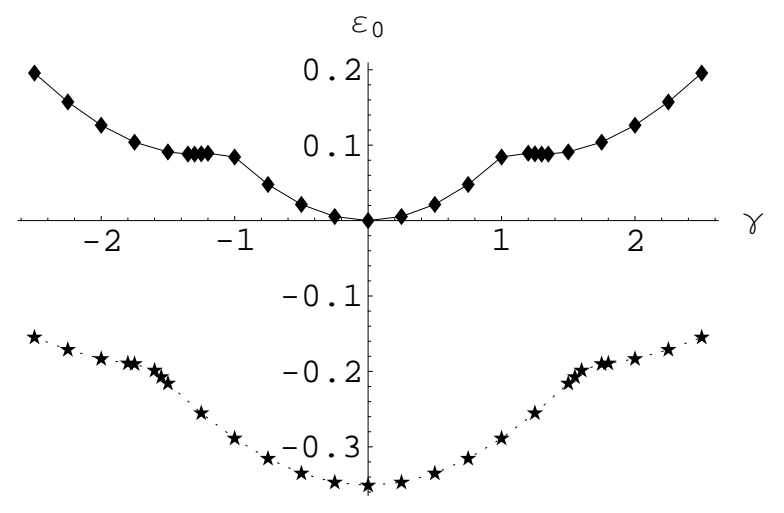

Fig. 6 


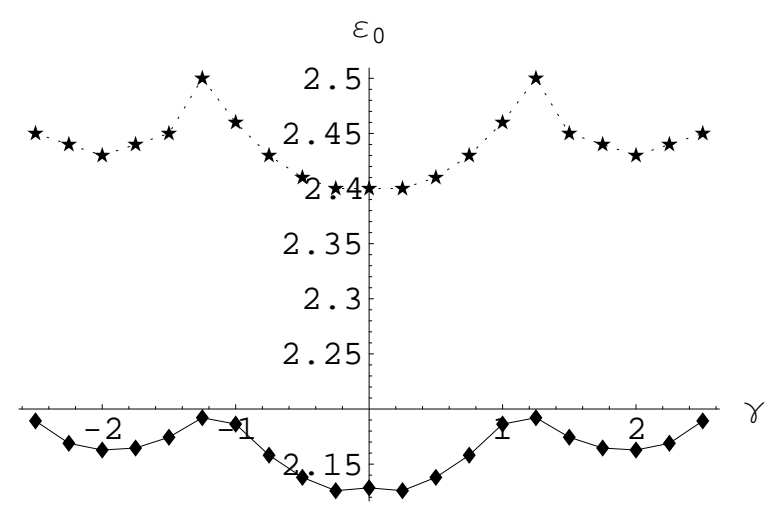

Fig. 7

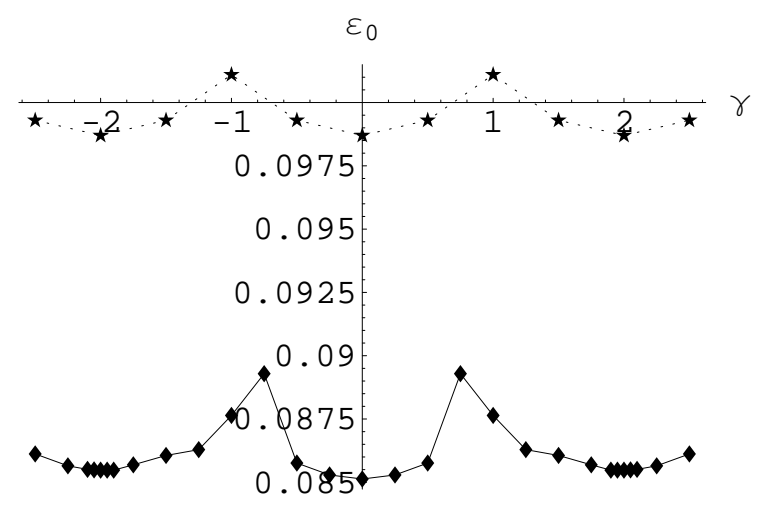

Fig. 8 